\title{
Spatio-Temporal EEG Models for Brain Interfaces
}

\author{
P. Gonzalez-Navarro ${ }^{\text {a }}$, M. Moghadamfalahi ${ }^{\mathrm{a}}$, M. Akcakaya $^{\mathrm{b}}$, D. Erdogmus ${ }^{\mathrm{a}}$ \\ ${ }^{a}$ Northeastern University, Boston, MA \\ ${ }^{b}$ University of Pittsburgh, Pittsburgh, PA
}

\begin{abstract}
Multichannel electroencephalography (EEG) is widely used in non-invasive brain computer interfaces (BCIs) for user intent inference. EEG can be assumed to be a Gaussian process with unknown mean and autocovariance, and the estimation of parameters is required for BCI inference. However, the relatively high dimensionality of the EEG feature vectors with respect to the number of labeled observations lead to rank deficient covariance matrix estimates. In this manuscript, to overcome ill-conditioned covariance estimation, we propose a structure for the covariance matrices of the multichannel EEG signals. Specifically, we assume that these covariances can be modeled as a Kronecker product of temporal and spatial covariances. Our results over the experimental data collected from the users of a letter-by-letter typing BCI show that with less number of parameter estimations, the system can achieve higher classification accuracies compared to a method that uses full unstructured covariance estimation. Moreover, in order to illustrate that the proposed Kronecker product structure could enable shortening the BCI calibration data collection sessions, using Cramer-Rao bound analysis on simulated data, we demonstrate that a model with structured covariance matrices will achieve the same estimation error as a model with no covariance structure using fewer labeled EEG observations.
\end{abstract}

Keywords: Structured covariance matrices, Kronecker product, brain computer interface, multichannel electroencephalogram (EEG), auto-regressive (AR) model, linear mixture.

\section{Introduction}

Electroencephalography (EEG)-based brain computer interfaces (BCIs) offer people with severe speech and physical impairments (SSPI) an alter- 
native communication method(1). Event related potentials (ERPs), steady state evoked potentials (SSVEP) or voluntarily controlled cortical potentials are commonly employed by the EEG-based BCIs to detect the user intent (2; 1; 3; 4; 5; 6; 7; 8).

In most EEG-based BCI systems, signal recorded from multiple channels along the scalp is assumed to be a Gaussian process with an unknown covariance and mean (9, 10, 11). In this setup a quadratic discriminant analysis (QDA) can achieve the minimum risk classification performance. To estimate the mean and the covariance for QDA, prior to operation of a BCI system, supervised data are collected through a calibration session (12; 13; 3, 14, 15). Typically, the multivariate EEG signal recorded from multiple channels in a time window is stacked into a feature vector, and feature vectors extracted from the supervised EEG data in such a fashion are used to estimate the covariance and the mean of the Gaussian process. In such an approach, due to the high dimensionality of the EEG feature vectors, an invertible estimation of covariance requires many supervised data samples; that is, a longer calibration session. However, a long calibration session is not always possible, since it might decrease the system practicality and lead to user frustration. In consequence, the estimated sample covariance is rank-deficient and over-fitted to the data (16, 17). In our earlier work, in the development of a language model assisted letter-by-letter typing BCI, which we call as RSVPKeyboard ${ }^{\mathrm{TM}}$, we have utilized regularized discriminant analysis (RDA) to overcome this issue (15, 18). In a classification setup, RDA applies regularization and shrinkage in the estimation of covariances (19). This approach has shown promising results (5; 18). However such an approach, ignores any inherent structure of covariance matrices and may require more labeled data for estimation compared to methods that impose a covariance structure.

In this manuscript, we hypothesize that imposing a Kronecker product (KP) structure on a covariance matrix of the EEG signal model will result in a smaller number of parameters to be estimated and hence, a smaller training set from a calibration session. This structure implies that the multichannel EEG signal is assumed to have a covariance matrix that is the KP of temporal and spatial covariances. This means that the KP structure assumes spatiotemporal stationarity over the multichannel EEG. In this paper, we describe two signal modeling approaches for the multichannel EEG signal which will lead to KP covariance structure under certain assumptions. In our approach, we use a spatio-temporal linear forward model from unknown 
brain sources to the EEG electrode sites to model the multichannel EEG. We also show that this is equivalent to using an auto-regressive moving average (ARMA) model over time and channels. Over this model, we show that under certain independence and stationarity assumptions the full covariance matrix can be factorized as a Kronecker product of temporal and spatial covariance matrices. We then introduce further temporal assumptions on the signal to achieve specific structures on temporal covariance matrix: (i) Toeplitz matrix structure, (ii) auto-regressive model with degree $1(\operatorname{AR}(1))$ structure, and (iii) identity matrix structure. These additional structures further decrease the number of parameters to be estimated. We also compute the Cramer-Rao bound (CRB) on covariance estimation error and use this as a performance metric to analyze the number of observations required to achieve a desired estimation accuracy; that is, the CRB indicates a calibration session length. We have collected data from 12 healthy participants using RSVPKeyboard ${ }^{\mathrm{TM}}$ and compared their typing performance under different covariance structures. Our results show that with much lower model complexity, the structured covariance assumption provides the same or better typing accuracy compared to a model with an unstructured covariance.

\section{RSVPKeyboard ${ }^{\mathrm{TM}}$}

In this section, we briefly describe the main components of RSVPKeyboard ${ }^{\mathrm{TM}}$. Interested readers could refer to (20) for further information on RSVPKeyboard ${ }^{\mathrm{TM}}$.

\subsection{Presentation Paradigms}

RSVPKeyboard ${ }^{\mathrm{TM}}$ employs three different presentation paradigms.

(i). Rapid Serial Visual Presentation (RSVP): is a paradigm for which a set of pseudo-randomly ordered stimuli are presented on prefixed location of the screen in a rapid serial manner. In RSVP each stimulus is a trial and a set of trials which have been presented with no time gap in between, is called a sequence. Every sequence contains only a single target stimulus.

(ii). Single Character Presentation (SCP): consists of sequential flashes of individual characters placed on a $R \times C$ matrix background in a pseudo-random order. In SCP each stimulus is a trial and a set of trials which have been presented with no time gap in between is called a sequence. Every sequence contains a single target stimulus. 
(iii). Row and Column Presentation ( $R C P)$ : consists of sequential flashes of sets of characters which are placed on a row or a column of a $R \times C$ matrix background in a pseudo-random order. In RCP each row or column is called a trial and a full set of rows and columns which are flashing with no time gap in between is called a sequence. Every sequence in RCP, contains two target stimuli.

\subsection{Probabilistic Inference Mechanism}

RSVPKeyboard $^{\mathrm{TM}}$ utilizes a maximum a-posteriori (MAP) inference for intent detection. Assume $\mathcal{D}$ as the dictionary of all candidates for user intent and $s \in \mathcal{D}$. We define $s^{*}$ as the random variable which represents the actual user intent and $\hat{s}$ is the system estimation. Then the decision rule is as follows:

$$
\hat{s}=\arg \max _{\mathbf{s} \in \mathcal{D}} P\left(s^{*}=\mathrm{s} \mid \mathcal{E} ; C\right)
$$

Here $\mathcal{E}$ represents the recorded EEG during the presentation of a sequence of symbols and $C$ demonstrates the non-EEG contextual evidences (for detailed information please see (3)). Prior to inference the system perform EEG prepossessing and feature extraction followed by a quadratic projection to improve classification performance.

\subsection{Signal Processing and Feature Extraction}

The EEG time signal is recorded from $N_{c h}$ channels along the scalp at a particular sampling rate (here $256 \mathrm{~Hz}$ ). We utilize a bandpass filter to increase the signal to noise ratio (SNR) and eliminate the slow drifts. Based on the high cut-off frequency of the filter, one can propose to reduce the sampling rate while preventing from aliasing. The multivariate EEG signals recorded from multiple channels in a time window of $[0, w) \mathrm{ms}$, from the onset of every trial, is assigned as the EEG features for that trial. We concatenate the multivariate EEG time samples to form the feature vector. Assume $\mathbf{v}^{i}[n]$, the multivariate measurement at the time instant $n$, defined as follows:

$$
\mathbf{v}^{i}[n]=\left[\begin{array}{llll}
v_{1}^{i}[n] & v_{2}^{i}[n] \ldots v_{N_{c h}}^{i}[n]
\end{array}\right]^{T} \in \mathbb{R}^{N_{c h}}
$$

where $v_{c h}^{i}[n]$ is the $n^{\text {th }}$ time sample recorded at channel $c h$ for trial $i$ (note that $n$ is measured with respect to each trial one-set time). Then we can 
define $\mathbf{y}^{i}$ as the feature vector for trial $i$ as follows:

$$
\mathbf{y}^{i}=\left[\mathbf{v}^{i}[1]^{T} \mathbf{v}^{i}[2]^{T} \ldots \mathbf{v}^{i}\left[N_{t}\right]^{T}\right]^{T} \in \mathbb{R}^{N_{c h} \cdot N_{t}}
$$

Here $N_{t}$ is the number of time samples in a window of $[0, w) \mathrm{ms}$.

We further perform a quadratic projection of these feature vectors on to a one dimensional evidence space. One can propose the quadratic discriminant analysis (QDA) to obtain a minimum expected classification risk for this projection. In QDA the log-likelihood of the observations can be defined as:

$$
l^{i}\left(\mathbf{y}^{i}\right)=\log \frac{f_{\mathcal{N}}\left(\mathbf{y}^{i} ; \boldsymbol{\mu}_{1}, \boldsymbol{\Sigma}_{1}\right) \pi_{1}}{f_{\mathcal{N}}\left(\mathbf{y}^{i} ; \boldsymbol{\mu}_{0}, \boldsymbol{\Sigma}_{0}\right) \pi_{0}}
$$

where $f_{\mathcal{N}}\left(\cdot, \boldsymbol{\mu}_{\phi}, \boldsymbol{\Sigma}_{\phi}\right)$ is the multivariate normal density function with mean $\boldsymbol{\mu}_{\phi}$, covariance $\boldsymbol{\Sigma}_{\phi}$, and prior $\pi_{\phi}$. This log-likelihood ratio $l^{i}\left(\mathbf{y}^{i}\right)$ from equation (4) is the EEG evidence for the $i^{\text {th }}$ trial where $\phi=1$ represents the target class and $\phi=0$ defines the non-target class.

In order to perform the projection, explained in (4) one needs to estimate the class conditional probability distributions of target $(\phi=1)$ and nontarget $(\phi=0)$ classes. RSVPKeyboard ${ }^{\mathrm{TM}}$ utilizes a set of supervised data collected in a calibration session, to estimate the class conditional probability distributions in maximum likelihood framework. Typically, a calibration session consists of 100 sequences of many trials. Before every sequence, the user is presented with a target stimulus which is expected to be the intent of the user during the presentation of that sequence.

The parameters of class conditional probability density functions (PDFs) $f_{\mathcal{N}}\left(\cdot, \boldsymbol{\mu}_{\phi}, \boldsymbol{\Sigma}_{\phi}\right)$ are estimated as follows:

$$
\begin{aligned}
\boldsymbol{\mu}_{\phi} & =\frac{1}{N_{\phi}} \sum_{i=1}^{N} \mathbf{y}^{i} \delta\left(\psi_{i}, \phi\right) \\
\boldsymbol{\Sigma}_{\phi} & =\frac{1}{N_{\phi}} \sum_{i=1}^{N}\left(\mathbf{y}^{i}-\boldsymbol{\mu}_{\phi}\right)\left(\mathbf{y}^{i}-\boldsymbol{\mu}_{\phi}\right)^{T} \delta\left(\psi_{i}, \phi\right)
\end{aligned}
$$

where $\psi_{i} \in\{0,1\}$ is the label of $\mathbf{y}^{i}, \delta(.,$.$) is the Kronecker delta function,$ $\phi \in\{0,1\}$ is the class label, $N$ represents the total number of observations and $N_{\phi}=\sum_{i=1}^{N} \delta\left(\psi_{i}, \phi\right)$ is the number of observations in class $\phi$.

Estimating an invertible covariance matrix $\boldsymbol{\Sigma}_{\phi}$ from equation (5) requires at least $N_{c h} \cdot N_{t}+1$ observations in each class. However, this can lead to 
extremely long calibration sessions which practically is not feasible. This issue can be relaxed by regularizing the estimated covariances as in regularized discriminant analysis (RDA) which is a generalization of QDA (19). RDA regularizes the class conditional covariance matrices in two steps of (i) shrinkage and (ii) regularization as presented in (6).

$$
\begin{aligned}
& \widehat{\boldsymbol{\Sigma}}_{\phi}(\lambda)=\frac{(1-\lambda) N_{\phi} \boldsymbol{\Sigma}_{\phi}+(\lambda) \sum_{\phi=0}^{1} N_{\phi} \boldsymbol{\Sigma}_{\phi}}{(1-\lambda) N_{\phi}+(\lambda) \sum_{\phi=0}^{1} N_{\phi}} \\
& \widehat{\boldsymbol{\Sigma}}_{\phi}(\lambda, \gamma)=(1-\gamma) \widehat{\boldsymbol{\Sigma}}_{\phi}(\lambda)+(\gamma) \frac{1}{p} \operatorname{tr}\left[\widehat{\boldsymbol{\Sigma}}_{\phi}(\lambda)\right] \mathbb{I}_{p}
\end{aligned}
$$

Here, $\lambda, \gamma \in[0,1]$ are the shrinkage and regularization parameters, $\operatorname{tr}[\cdot]$ is the trace operator and $\mathbb{I}_{p}$ is an identity matrix of size $p \times p$. In our system, we typically utilize $\mathrm{k}$-fold cross-validation to choose the optimal values of hyper-parameters $\lambda$ and $\gamma$.

In addition, as we show in this manuscript, an EEG signal model under specific assumptions can factorize the class conditional covariance matrices as the following Kronecker product.

$$
\Sigma_{\phi}=\Sigma_{t}^{\phi} \otimes \Sigma_{c}^{\phi}
$$

In this equation $\boldsymbol{\Sigma}_{t}^{\phi}$ and $\boldsymbol{\Sigma}_{c}^{\phi}$ are the temporal and spatial covariance matrices, respectively. Such a structure can reduce the number of parameters to be estimated dramatically and results in an invertible covariance estimate from the calibration data. Next, we describe the models that we propose for the EEG the signal and introduce different covariance structures.

\section{Signal Models and Covariance Matrix Structures}

In this section, we first model the multichannel EEG as a linear forward model from unknown brain sources and then show that this modeling approach is equivalent to using an ARMA model over the multichannel EEG under certain assumptions. Linear forward model describes the EEG recorded from $N_{c h}$ channels along the scalp as a linear combination of signals originated from $N_{s}$ brain sources. In Section 3.1, we first show that certain assumptions on this linear forward model leads to a Kronecker product structure as described above. We then model the brain source signals as: (i) a stationary signal, (ii) an auto-regressive process of order one $\mathrm{AR}(1)$ and (iii) 
an auto-regressive process of order zero $\mathrm{AR}(0)$ to impose more structure on the temporal covariance and hence reduce the number of parameters even further. Then in Section 3.2, we show that an ARMA model over the multichannel EEG also leads to the same spatiotemporal KP structure under certain stationarity assumptions imposed over time and EEG channels.

\subsection{Linear Forward Model}

We propose to define the multichannel EEG signal as a linear combination of unknown brain sources. Assume

$$
\mathbf{s}^{i}[n]=\left[\begin{array}{llll}
s_{1}^{i}[n] & s_{j}^{i}[n] \ldots & s_{N_{s}}^{i}[n]
\end{array}\right]^{T} \in \mathbb{R}^{N_{s}}
$$

as the vector of brain source signals, where $N_{s}$ is the unknown number of brain sources and $s_{j}^{i}[n]$ is the generated signal from source $j$ at time sample $n$ for the $i^{\text {th }}$ trial. Then the linear model can be defined as:

$$
\mathbf{v}^{i}[n]=\mathbf{H s}^{i}[n] \in \mathbb{R}^{N_{c h}}
$$

where

$$
\mathbf{H}=\left[\begin{array}{ccc}
h_{1,1} & \ldots & h_{1, N_{s}} \\
h_{2,1} & \ldots & h_{2, N_{s}} \\
\vdots & & \vdots \\
h_{N_{c h}, 1} & \ldots & h_{N_{c h}, N_{s}}
\end{array}\right] \in \mathbb{R}^{N_{c h} \times N_{s}}
$$

is the forward combination matrix for which, every $h_{c, j}, \forall 0<c \leq N_{c h}, 0<$ $j \leq N_{s}$ is a real coefficient. In this manuscript we assume that the number of brain sources is higher than or equal to the number of channels, which means that the matrix $\mathbf{H}$ is a full row rank matrix.

We then define $\mathbf{x}^{i}$ as the feature vector of brain sources such that

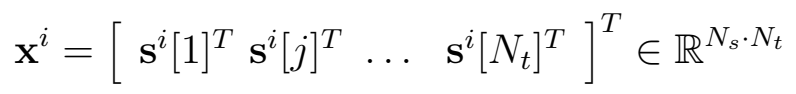

In this model, assuming that the matrix $\mathbf{H}$ is constant through time, the linear model of the EEG feature vector, $\mathbf{y}^{i}$, which is defined in equation (3), becomes

$$
\mathbf{y}^{i}=\mathbf{H}_{f} \mathbf{x}^{i}
$$

where $\mathbf{H}_{f}$ is a time invariant block diagonal matrix of dimensions $\left(N_{t} N_{c h}\right) \times$ 
$\left(N_{t} N_{s}\right)$ and the $N_{c h} \times N_{s}$ blocks are equal to the matrix $\mathbf{H}$.

$$
\mathbf{H}_{f}=\left[\begin{array}{cccc}
\mathbf{H} & 0 & \cdots & 0 \\
0 & \mathbf{H} & 0 & 0 \\
\vdots & 0 & \ddots & 0 \\
0 & 0 & 0 & \mathbf{H}
\end{array}\right] \in \mathbb{R}^{\left(N_{t} \cdot N_{c h}\right) \times\left(N_{t} \cdot N_{s}\right)}
$$

We further assume that $\mathbf{s}^{1}[n], \mathbf{s}^{2}[n], \cdots, \mathbf{s}^{i}[n], \cdots \sim \mathcal{N}_{N_{s}}\left(\boldsymbol{\mu}_{\mathbf{s}}[n], \boldsymbol{\Sigma}_{\mathbf{s}}[n, n]\right)$ are i.i.d. samples, where

$$
\begin{array}{ll}
\boldsymbol{\mu}_{\mathbf{s}}[n] & =E[\mathbf{s}[n]] \\
\boldsymbol{\Sigma}_{\mathbf{s}}[n, n] & =\operatorname{Cov}\left[\mathbf{s}^{i}[n], \mathbf{s}^{i}[n]\right]
\end{array}
$$

Accordingly, from (8) we can define $\mathbf{v}^{i}[n] \sim \mathcal{N}_{N_{s}}\left(\boldsymbol{\mu}_{\mathbf{v}}[n], \boldsymbol{\Sigma}_{\mathbf{v}}[n, n]\right)$ for which we have:

$$
\begin{aligned}
\boldsymbol{\mu}_{\mathbf{v}}[n] & =E[\mathbf{v}[n]] \\
\boldsymbol{\Sigma}_{\mathbf{v}}[n, n] & =\operatorname{Cov}\left[\mathbf{v}^{i}[n], \mathbf{v}^{i}[n]\right]=\mathbf{H} \boldsymbol{\Sigma}_{\mathbf{s}}[n, n] \mathbf{H}^{T}
\end{aligned}
$$

Therefore, under these assumptions, one should consider that $\mathbf{x}^{i} \sim \mathcal{N}_{N_{t} \cdot N_{s}}\left(\boldsymbol{\mu}_{\mathbf{x}}, \boldsymbol{\Sigma}_{\mathbf{x}}\right)$ and also $\mathbf{y}^{i} \sim \mathcal{N}_{N_{c h} \cdot N_{s}}\left(\boldsymbol{\mu}_{\mathbf{y}}, \boldsymbol{\Sigma}_{\mathbf{y}}\right)$.

\subsubsection{Assumptions on the Spatial Characteristics of the Brain Sources}

In our approach, the factorization in (7) achieved using the following assumptions. We first assume that the unknown brain sources are statistically independent from each other. This implies that $\boldsymbol{\Sigma}_{\mathbf{s}}[m, n] \quad \forall 0 \leq m, n \leq N_{t}$ is a diagonal matrix.

$$
\boldsymbol{\Sigma}_{\mathbf{s}}[m, n]=\left[\begin{array}{cccc}
c_{s}[m, n]_{1,1} & 0 & \cdots & 0 \\
0 & c_{s}[m, n]_{2,2} & 0 & 0 \\
\vdots & 0 & \ddots & 0 \\
0 & 0 & 0 & c_{s}[m, n]_{N_{s}, N_{s}}
\end{array}\right]
$$

where $c_{s}[m, n]_{j, j}$ is a scalar value which represents the correlation between the $m^{\text {th }}$ and $n^{\text {th }}$ time samples of the $j^{\text {th }}$ brain source. Furthermore, assuming 
that all brain sources have the same covariance structure; we have:

$$
\boldsymbol{\Sigma}_{\mathbf{s}}[m, n]=\left[\begin{array}{cccc}
c_{s}[m, n] & 0 & \cdots & 0 \\
0 & c_{s}[m, n] & 0 & 0 \\
\vdots & 0 & \ddots & 0 \\
0 & 0 & 0 & c_{s}[m, n]
\end{array}\right]=c_{s}[m, n] \cdot \mathbb{I}_{N_{s}}
$$

Then following equation $(11)$, we can write $\boldsymbol{\Sigma}_{\mathbf{y}}$ as follows:

$$
\begin{aligned}
& \boldsymbol{\Sigma}_{\mathbf{y}}=\mathbf{H}_{f} \boldsymbol{\Sigma}_{\mathbf{x}} \mathbf{H}_{f}^{T}=\left[\begin{array}{ccc}
\mathbf{H} \boldsymbol{\Sigma}_{\mathbf{s}}[1,1] \mathbf{H}^{T} & \ldots & \mathbf{H} \boldsymbol{\Sigma}_{\mathbf{s}}\left[1, N_{t}\right] \mathbf{H}^{T} \\
\mathbf{H} \boldsymbol{\Sigma}_{\mathbf{s}}[2,1] \mathbf{H}^{T} & \ldots & \mathbf{H} \boldsymbol{\Sigma}_{\mathbf{s}}\left[2, N_{t}\right] \mathbf{H}^{T} \\
\vdots & & \vdots \\
\mathbf{H} \boldsymbol{\Sigma}_{\mathbf{s}}\left[N_{t}, 1\right] \mathbf{H}^{T} & \ldots & \mathbf{H} \boldsymbol{\Sigma}_{\mathbf{s}}\left[N_{t}, N_{t}\right] \mathbf{H}^{T}
\end{array}\right] \\
& =\left[\begin{array}{ccc}
\mathbf{H} c_{s}[1,1] \mathbb{I}_{N_{s}} \mathbf{H}^{T} & \ldots & \mathbf{H} c_{s}\left[1, N_{t}\right] \mathbb{I}_{N_{s}} \mathbf{H}^{T} \\
\mathbf{H} c_{s}[2,1] \mathbb{I}_{N_{s}} \mathbf{H}^{T} & \ldots & \mathbf{H} c_{s}\left[2, N_{t}\right] \mathbb{I}_{N_{s}} \mathbf{H}^{T} \\
\vdots & & \vdots \\
\mathbf{H} c_{s}\left[N_{t}, 1\right] \mathbb{I}_{N_{s}} \mathbf{H}^{T} & \ldots & \mathbf{H} c_{s}\left[N_{t}, N_{t}\right] \mathbb{I}_{N_{s}} \mathbf{H}^{T}
\end{array}\right] \\
& =\left[\begin{array}{ccc}
c_{s}[1,1] & \ldots & c_{s}\left[1, N_{t}\right] \\
c_{s}[2,1] & \ldots & c_{s}\left[2, N_{t}\right] \\
\vdots & & \vdots \\
c_{s}\left[N_{t}, 1\right] & \ldots & c_{s}\left[N_{t}, N_{t}\right]
\end{array}\right] \otimes \mathbf{H H}^{T}
\end{aligned}
$$

Note that equation (17) is in the same factorization form as (7). Throughout this manuscript the matrix $\mathbf{H H}^{T} \in \mathbb{R}^{N_{c h} \times N_{c h}}$ is assumed as the spatial covariance matrix $\Sigma_{c h}$. For this spatial matrix to be invertible, we assume that the number of the brain sources is at least the same as the number of the channels. The temporal covariance matrix is defined as follows:

$$
\Sigma_{t}=\left[\begin{array}{ccc}
c_{s}[1,1] & \ldots & c_{s}\left[1, N_{t}\right] \\
c_{s}[2,1] & \ldots & c_{s}\left[2, N_{t}\right] \\
\vdots & & \vdots \\
c_{s}\left[N_{t}, 1\right] & \ldots & c_{s}\left[N_{t}, N_{t}\right]
\end{array}\right] \in \mathbb{R}^{N_{t} \times N_{t}}
$$


Accordingly we model the full covariance matrix of the EEG feature vectors as a Kronecker product of a spatial and temporal covariances matrices

$$
\Sigma_{\mathbf{y}}=\Sigma_{t} \otimes \Sigma_{c h}
$$

Under this covariance structure the number of parameters that need to estimated is:

$$
N_{p_{1}}=\frac{N_{c h}\left(N_{c h}+1\right)}{2}+\frac{N_{t}\left(N_{t}+1\right)}{2}
$$

Different assumptions for specific temporal covariance structures are described next.

\subsubsection{Assumptions on the Temporal Characteristics of the Brain Sources}

Brain sources as stationary processes:

If we assume that the brain sources are stationary in time with $\boldsymbol{\Sigma}_{\mathbf{s}}[m, n]=$ $\Sigma_{\mathbf{s}}[m-n]$; that is, we have $c_{s}[m, n]=c_{s}[m-n]$. Then, the temporal covariance matrix becomes a Toeplitz matrix

$$
\Sigma_{t}=\left[\begin{array}{ccc}
c_{s}[0] & \ldots & c_{s}\left[N_{t}-1\right] \\
& \ddots & \vdots \\
c_{s}\left[N_{t}-1\right] & \ldots & c_{s}[0]
\end{array}\right]
$$

and accordingly the full covariance matrix is characterized by

$$
N_{p_{2}}=\frac{N_{c h}\left(N_{c h}+1\right)}{2}+N_{t}
$$

number of parameters.

\section{Brain sources as an Autoregressive (AR) model:}

The $p^{\text {th }}$ order AR model of the multivariate signal $\mathbf{s}^{i}[n]$ can be written as

$$
\mathbf{s}^{i}[n]=\sum_{k=1}^{p} A_{k} \mathbf{s}^{i}[n-k]+\boldsymbol{e}^{i}[n]
$$

where $A_{k}$ represents the $N_{s} \times N_{s}$ weight matrix at time lag $k$, and $\boldsymbol{e}^{i}[n]$ is zero mean additive Gaussian noise at time $n$ that is independent from the sources and independent and identically distributed in time. Further, we assume that not only the brain sources are statistically independent but also 
the AR model among all the sources is invariant. Then, we rewrite (23) as

$$
\mathbf{s}^{i}[n]=\sum_{k=1}^{p} a_{k} \cdot \mathbf{s}^{i}[n-k]+\boldsymbol{e}^{i}[n]
$$

where $a_{k}$ is a scalar weight for the signal at time lag $k$. In our approach, we consider $p=1$ in this section and $p=0$ later in Section III.C.3. When we have $p=1,24$ becomes

$$
\mathbf{s}_{n}^{i}=a_{1} \cdot \mathbf{s}^{i}[n-1]+\boldsymbol{e}^{i}[n]
$$

Then, assuming that

$$
\mathbf{s}[0] \sim \mathcal{N}_{N_{s}}\left(\boldsymbol{\mu}_{\mathbf{s}}[0], \boldsymbol{\Sigma}_{\mathbf{s}}[0,0]\right)
$$

from 25, we define the mean and the covariance as

$$
\mathbf{s}^{i}[n]=\left(a_{1}\right)^{n} \cdot \mathbf{s}[0] \Rightarrow E\left[\mathbf{s}^{i}[n]\right]=\left(a_{1}\right)^{n} \cdot \boldsymbol{\mu}_{\mathbf{s}}[0]
$$

and for $m \leq n$,

$$
\begin{aligned}
\boldsymbol{\Sigma}_{\mathbf{s}}[m, n] & =E\left\{\mathbf{s}^{i}[n] \mathbf{s}^{i}[m]^{T}\right\}-E\left\{\mathbf{s}^{i}[n]\right\} E\left\{\mathbf{s}^{i}[m]\right\}^{T} \\
& =a_{1}^{|(n-m)|} \cdot\left(E\left\{\mathbf{s}^{i}[m] \mathbf{s}^{i}[m]^{T}\right\}-\boldsymbol{\mu}_{\mathbf{s}}[m] \boldsymbol{\mu}_{\mathbf{s}}[m]^{T}\right) \\
& =a_{1}^{|(n-m)|} \cdot \boldsymbol{\Sigma}_{\mathbf{s}}[m, m] \\
& =a_{1}^{|(n-m)|} \cdot \boldsymbol{\Sigma}_{\mathbf{s}}[0,0]
\end{aligned}
$$

One should note that $\boldsymbol{\Sigma}_{\mathbf{s}}[n, m]=\boldsymbol{\Sigma}_{\mathbf{s}}[m, n]$ and $\boldsymbol{\Sigma}_{\mathbf{s}}[n, m]$ is a symetric matrix. Then by substituting (28) in (10), the covariance matrix of the brain source 
signals $\boldsymbol{\Sigma}_{\mathbf{x}}$ becomes

$$
\begin{aligned}
\boldsymbol{\Sigma}_{\mathbf{x}}= & {\left[\begin{array}{ccc}
\boldsymbol{\Sigma}_{\mathbf{s}}[1,1] & \ldots & \boldsymbol{\Sigma}_{\mathbf{s}}\left[1, N_{t}\right] \\
\boldsymbol{\Sigma}_{\mathbf{s}}[2,1] & & \boldsymbol{\Sigma}_{\mathbf{s}}\left[2, N_{t}\right] \\
\vdots & & \vdots \\
& & \\
\boldsymbol{\Sigma}_{\mathbf{s}}\left[N_{t}, 1\right] & \ldots & \boldsymbol{\Sigma}_{\mathbf{s}}\left[N_{t}, N_{t}\right]
\end{array}\right] } \\
= & {\left[\begin{array}{cccc}
1 & a_{1}^{(1)} & \ldots & a_{1}^{\left(N_{t}-1\right)} \\
a_{1}^{(1)} & 1 & \ldots & a_{1}^{\left(N_{t}-2\right)} \\
\vdots & \vdots & \ddots & \vdots \\
a_{1}^{\left(N_{t}-1\right)} & a_{1}^{\left(N_{t}-2\right)} & \ldots & 1
\end{array}\right] \otimes \boldsymbol{\Sigma}_{\mathbf{s}}[0,0] }
\end{aligned}
$$

Considering the assumptions introduced in subsection (3.1.1), we have $\boldsymbol{\Sigma}_{\mathbf{s}}[m, n]=$ $c_{s}[m, n] \cdot \mathbb{I}_{N_{s}} \forall m, n$, then from equation (28), we have:

$$
\begin{gathered}
\boldsymbol{\Sigma}_{\mathbf{s}}[0,0]=c_{s}[0] \cdot \mathbb{I}_{N_{s}} \\
\boldsymbol{\Sigma}_{\mathbf{s}}[m, n]=a_{1}^{(|n-m|)} \cdot \boldsymbol{\Sigma}_{\mathbf{s}}[0]=a_{1}^{(|n-m|)} \cdot c_{s}[0] \cdot \mathbb{I}_{N_{s}}
\end{gathered}
$$

Using the linear forward model and the equation (31), we compute the full covariance matrix of $\boldsymbol{\Sigma}_{\mathbf{y}}$ as:

$$
\begin{aligned}
& \boldsymbol{\Sigma}_{\mathbf{y}}=\mathbf{H}_{f} \boldsymbol{\Sigma}_{\mathbf{x}} \mathbf{H}_{f}^{T} \\
& =\left[\begin{array}{ccc}
\mathbf{H} c_{s}[0] \mathbb{I}_{N_{s}} \mathbf{H}^{T} & \ldots & \mathbf{H} a_{1}^{\left(N_{t}-1\right)} c_{s}[0] \mathbb{I}_{N_{s}} \mathbf{H}^{T} \\
\mathbf{H} a_{1}^{(1)} c_{s}[0] \mathbb{I}_{N_{s}} \mathbf{H}^{T} & \ldots & \mathbf{H} a_{1}^{\left(N_{t}-2\right)} c_{s}[0] \mathbb{I}_{N_{s}} \mathbf{H}^{T} \\
\vdots & & \vdots \\
\mathbf{H} a_{1}^{\left(N_{t}-1\right)} c_{s}[0] \mathbb{I}_{N_{s}} \mathbf{H}^{T} & \ldots & \mathbf{H} c_{s}[0] \mathbb{I}_{N_{s}} \mathbf{H}^{T}
\end{array}\right] \\
& =c_{s}[0] \cdot\left[\begin{array}{cccc}
1 & a_{1}^{(1)} & \ldots & a_{1}^{\left(N_{t}-1\right)} \\
a_{1}^{(1)} & 1 & \ldots & a_{1}^{\left(N_{t}-2\right)} \\
\vdots & \vdots & \ddots & \vdots \\
a_{1}^{\left(N_{t}-1\right)} & a_{1}^{\left(N_{t}-2\right)} & \ldots & 1
\end{array}\right] \otimes \mathbf{H H}^{T}
\end{aligned}
$$


where $\mathbf{H H}^{T}$ is the spatial covariance matrix and the temporal covariance matrix is defined as follows

$$
\boldsymbol{\Sigma}_{t}=c_{s}[0] \cdot\left[\begin{array}{cccc}
1 & a_{1}^{(1)} & \ldots & a_{1}^{\left(N_{t}-1\right)} \\
a_{1}^{(1)} & 1 & \ldots & a_{1}^{\left(N_{t}-2\right)} \\
\vdots & \vdots & \ddots & \vdots \\
a_{1}^{\left(N_{t}-1\right)} & a_{1}^{\left(N_{t}-2\right)} & \ldots & 1
\end{array}\right]
$$

Where $c_{s}[0]$ is a constant value which is absorbed in the spatial covariance. Therefore, the $c_{s}[0]$ in equation 33 is estimated through the estimation of the spatial covariance and the temporal parameters in $N_{p} 3$ is then equal to 1. Under these assumptions the full covariance matrix can be fully characterized by:

$$
N_{p_{3}}=\frac{N_{c h}\left(N_{c h}+1\right)}{2}+1
$$

number of parameters.

\section{Brain sources as temporally independent processes:}

Under this assumption, we set $p=0$ in equation (24), then $\boldsymbol{\Sigma}_{\mathbf{s}}[m, n]=$ $\mathbf{0} \forall m \neq n$ and the temporal covariance $\boldsymbol{\Sigma}_{t}$ is

$$
\Sigma_{t}=I_{N_{t}}
$$

This structure requires:

$$
N_{p_{4}}=\frac{N_{c h}\left(N_{c h}+1\right)}{2}
$$

number of parameters to characterize the full covariance.

Note that in this section, one can observe that the number of parameters to be estimated to characterize the covariance matrix decreases as more restricted structures are assumed on the EEG signal model. Assume $N_{p_{0}}$ as the number of parameters of the non-structured full covariance, then:

$$
N_{p_{0}}=\frac{N_{c h} N_{t}\left(N_{c h} N_{t}+1\right)}{2}>N_{p_{1}}>N_{p_{2}}>N_{p_{3}}>N_{p_{4}}
$$




\subsection{ARMA model for the multicahnnel EEG signal}

We define an ARMA model for the multichannel EEG corresponding to the $i^{\text {th }}$ trial, $\mathbf{v}^{i}[n]$ as defined in (2), as follows:

$$
\mathbf{v}^{i}[n]=\sum_{k=1}^{p} R_{k} \mathbf{v}^{i}[n-k]+\sum_{j=0}^{q} b_{j} \mathbf{w}^{i}[n-j]
$$

In (38), $R_{k}$ represents the $N_{c h} \times N_{c h}$ signal weight matrix at lag $k, b_{j}$ is an scalar weight for noise at lag $j$ and $\mathbf{w}[n]$ represents multivariate wide sense stationary Gaussian noise for the $n^{\text {th }}$ time sample that is independent of $\mathbf{v}^{i}[n]$.

\subsubsection{Assumptions on the Spatiotemporal Characteristics of the EEG signal}

If we assume that the EEG signals among the channels is stationary. Then one can write $(38)$ as:

$$
\mathbf{v}^{i}[n]=\sum_{k=1}^{p} r_{k} \cdot \mathbf{v}^{i}[n-k]+\sum_{j=0}^{q} b_{j} \mathbf{w}^{i}[n-j]
$$

in which $r_{k}$ is an scalar weight of time for the signal at time lag $k$. Now lets further assume $p=1$ and $b_{j}=1$ for $j=0$ and $b_{j}=0 \forall j=1 \ldots q$ such that $\mathbf{w}^{i}$ represents the wide sense stationary Gaussian noise which is independent than the previous measurement $\mathbf{v}^{i}[n-1]$. Accordingly we can write:

$$
\mathbf{v}^{i}[n]=r_{1} \cdot \mathbf{v}^{i}[n-1]+\mathbf{w}^{i}[n]
$$

Lets assume $m<n$ and with wide-sense stationarity assumption over channels we will have:

$$
\begin{aligned}
\boldsymbol{\Sigma}_{\mathbf{v}}[m, n] & =E\left\{\mathbf{v}^{i}[n] \mathbf{v}^{i}[m]^{T}\right\}-E\left\{\mathbf{v}^{i}[n]\right\} E\left\{\mathbf{v}^{i}[m]\right\}^{T} \\
& =r_{1}^{(n-m)} \cdot\left(E\left\{\mathbf{v}^{i}[m] \mathbf{v}^{i}[m]^{T}\right\}-\boldsymbol{\mu}_{\mathbf{v}[m]} \boldsymbol{\mu}_{\mathbf{v}[m]}^{T}\right) \\
& =r_{1}^{(n-m)} \cdot \boldsymbol{\Sigma}_{\mathbf{v}}[m, m] \\
& =r[|n-m|] \cdot \boldsymbol{\Sigma}_{\mathbf{v}}[0,0] \\
& \text { where } r[|n-m|]=r_{1}^{(|n-m|)}
\end{aligned}
$$

According to definition of the multichannel EEG signal in (3) one can define 
the covariance matrix of $\mathbf{y}$ as:

$$
\begin{aligned}
\boldsymbol{\Sigma}_{\mathbf{y}}= & {\left[\begin{array}{ccc}
\boldsymbol{\Sigma}_{\mathbf{v}}[1,1] & \ldots & \boldsymbol{\Sigma}_{\mathbf{v}}\left[1, N_{t}\right] \\
\boldsymbol{\Sigma}_{\mathbf{v}}[2,1] & & \boldsymbol{\Sigma}_{\mathbf{v}}\left[2, N_{t}\right] \\
\vdots & & \vdots \\
& & \\
\boldsymbol{\Sigma}_{\mathbf{v}}\left[N_{t}, 1\right] & \ldots & \boldsymbol{\Sigma}_{\mathbf{v}}\left[N_{t}, N_{t}\right]
\end{array}\right] } \\
= & {\left[\begin{array}{ccc}
r[0] & \ldots & r\left[N_{t}-1\right] \\
r[1] & & r\left[N_{t}-2\right] \\
\vdots & & \vdots \\
r\left[N_{t}-1\right] & \ldots & r[0]
\end{array}\right] \otimes \boldsymbol{\Sigma}_{\mathbf{v}}[0,0] }
\end{aligned}
$$

where $\boldsymbol{\Sigma}_{\mathbf{v}}[0,0]$ is the spatial covariance matrix defined over the channels. Note here that while writing (42), we utilize the form that we obtain in (41). Then, following this representation given in 42 we generalize 40 such that

$$
\begin{gathered}
\mathbf{v}^{i}[n]=r[n, m] \cdot \mathbf{v}^{i}[n-1]+\mathbf{w}^{i}[n] \\
n=1 \ldots N_{t} \\
m=1 \ldots N_{t}
\end{gathered}
$$

Then we can show that

$$
\boldsymbol{\Sigma}_{\mathbf{y}}=\left[\begin{array}{ccc}
\boldsymbol{\Sigma}_{\mathbf{v}}[1,1] & \ldots & \boldsymbol{\Sigma}_{\mathbf{v}}\left[1, N_{t}\right] \\
\boldsymbol{\Sigma}_{\mathbf{v}}[2,1] & & \boldsymbol{\Sigma}_{\mathbf{v}}\left[2, N_{t}\right] \\
\vdots & & \vdots \\
& & \\
& & \\
\boldsymbol{\Sigma}_{\mathbf{v}}\left[N_{t}, 1\right] & \ldots & \boldsymbol{\Sigma}_{\mathbf{v}}\left[N_{t}, N_{t}\right]
\end{array}\right] \otimes \boldsymbol{\Sigma}_{\mathbf{v}}[0,0]
$$

Note that when the number of the brain sources is at least the same as number of channels (17) is equivalent to (44). If we assume the EEG signal 
is independent in time which means that $r[|n-m|]=0$ for all $m \neq n$. Then we have:

$$
\boldsymbol{\Sigma}_{\mathbf{y}}=r[0] \cdot \mathbf{I}_{N_{t}} \otimes \boldsymbol{\Sigma}_{\mathbf{v}}[0,0]
$$

Here $\mathbf{I}_{N_{t}}$ is an $N_{t} \times N_{t}$ identity matrix.

\section{Covariance Estimation Flip-Flop Algorithm}

In the proposed approach, we estimate the parameters of the covariance matrices using maximum likelihood estimation. Given a set of $N$ i.i.d. $\mathbf{y}^{1}, \mathbf{y}^{2}, \ldots, \mathbf{y}^{i}, \ldots \mathbf{y}^{N}$ samples from a zero mean multivariate Gaussian distribution with a Kronecker product covariance structure, the likelihood function is defined as

$$
L\left(\overline{\mathbf{Y}}, \boldsymbol{\Sigma}_{t} \otimes \boldsymbol{\Sigma}_{c h}\right)=\frac{\exp \left(-\sum_{i=1}^{N}\left(\mathbf{y}^{i}\right)^{T}\left(\boldsymbol{\Sigma}_{t}^{-1} \otimes \boldsymbol{\Sigma}_{c h}^{-1}\right) \mathbf{y}^{i}\right)}{\pi^{N_{t} \cdot N_{c h}}\left|\boldsymbol{\Sigma}_{t}^{h} \otimes \boldsymbol{\Sigma}_{c h}\right|^{N_{t} \cdot N_{c h}}}
$$

Using (46), we iterate between the estimation of the temporal and spatial components. Define the multivariate EEG time feature vector $\mathbf{t}_{c h}^{i}$ recorded from the $c h^{\text {th }}$ channel, as follows:

$$
\mathbf{t}_{c h}^{i}=\left[t_{c h}^{i}[1] t_{c h}^{i}[2] \ldots t_{c h}^{i}\left[N_{t}\right]\right]^{T} \in \mathbb{R}^{N_{t}}
$$

where $t_{c h}^{i}[n]$ is the $n^{\text {th }}$ time sample recorded at channel $c h$ for the $i^{\text {th }}$ trial. According to the definitions of the EEG feature vector $\mathbf{y}^{i}$ in Section 2.3 . $\mathbf{t}_{c h}^{i}$ represents the multivariate $N_{t}$ time measurements at one channel $c h$ of the feature vector $\mathbf{y}^{i}$. The maximum-likelihood estimate of the temporal covariance matrix for a fixed estimate of the spatial covariance matrix is:

$$
\widehat{\Sigma}_{t}=\frac{1}{N \cdot N_{c h}} \sum_{i=1}^{N} \sum_{j=1}^{N_{c h}} \sum_{p=1}^{N_{c h}} \sigma_{j p}^{c h} \mathbf{t}_{p}^{i}\left(\mathbf{t}_{j}^{i}\right)^{T}
$$

where $\sigma_{j p}^{c h}$ is the $(j, p)$ th element of the estimate of $\boldsymbol{\Sigma}_{c h}^{-1}$. Similarly, for a fixed estimate of the temporal covariance matrix, we compute an estimate for the spatial covariance matrix as

$$
\widehat{\mathbf{\Sigma}}_{c h}=\frac{1}{N \cdot N_{c h}} \sum_{i=1}^{N} \sum_{j=1}^{N_{t}} \sum_{p=1}^{N_{t}} \sigma_{j p}^{t} \mathbf{v}^{i}[p] \mathbf{v}^{i}[j]^{T}
$$




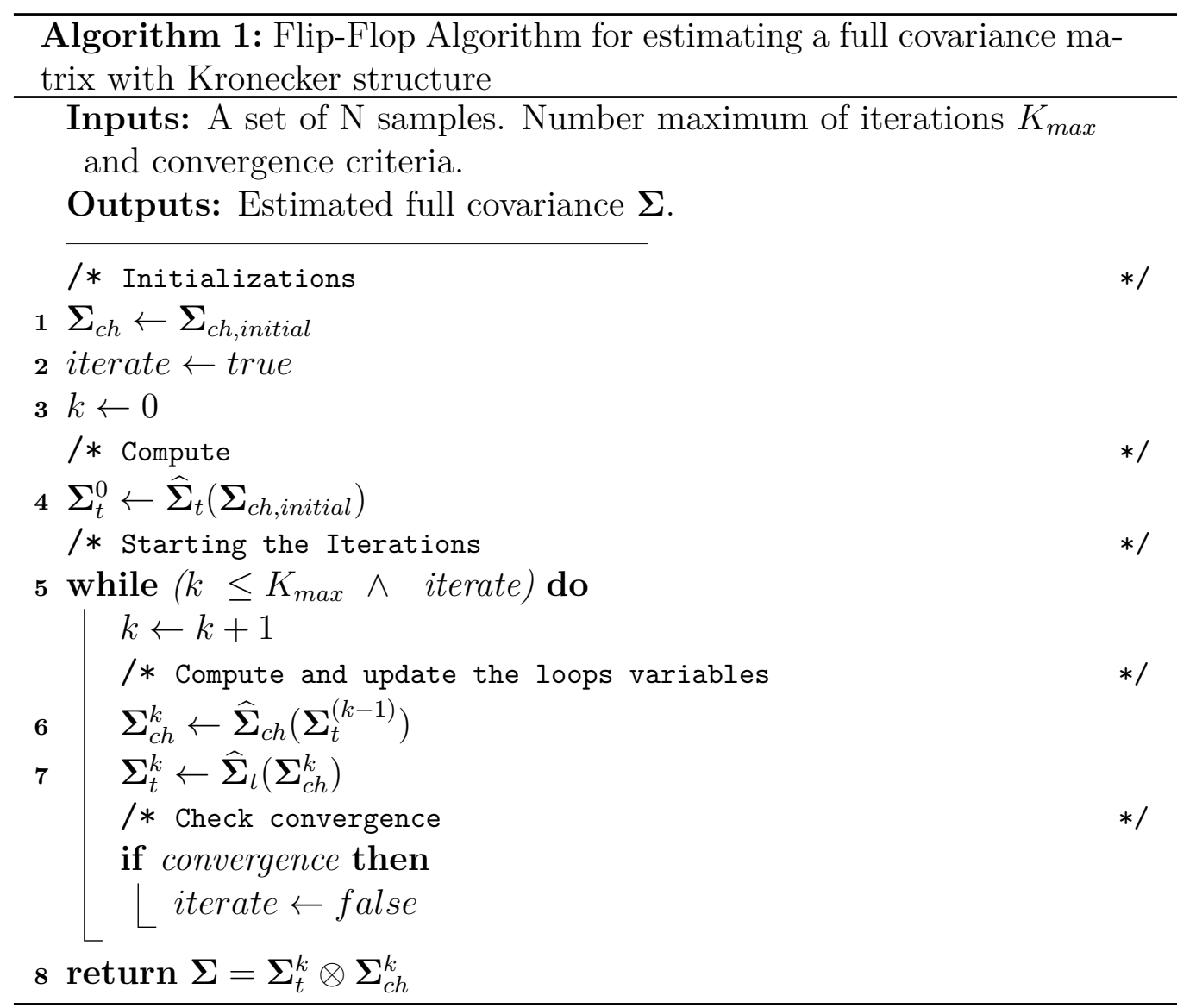

where $\sigma_{j p}^{t}$ is the $(j, p)$ th element of the estimate of $\boldsymbol{\Sigma}_{t}^{-1}$, and $\mathbf{v}^{i}[j]$ is the multivariate EEG feature vector according to (2) of Section 2.3.

This iterative approach is called the Flip-Flop algorithm and it is summarized in Algorithm (1). The Flip-Flop algorithm was shown to converge to the maximum likelihood estimate of the full covariance matrix (21).

The covariance estimates computed through the Flip-Flop algorithm are ambiguous up to a scaling factor because:

$$
\boldsymbol{\Sigma}=\boldsymbol{\Sigma}_{t} \otimes \boldsymbol{\Sigma}_{c h}=\gamma \cdot \boldsymbol{\Sigma}_{t} \otimes \gamma^{-1} \cdot \boldsymbol{\Sigma}_{c h}
$$

Hence, for estimating the parameters of the covariance matrices in the cases of general Kronecker-product structure, Kronecker-product with Toeplitz temporal covariance and Kronecker-product with AR(1) temporal covariance, we 
normalize the spatial covariance matrix to have the determinant of one, at each iteration. Moreover, for estimating the structured covariance matrix with the diagonal temporal covariance structure, we fix the temporal covariance as an identity matrix and then we perform a one-time estimation of the spatial covariance matrix using the Flip-Flop algorithm. To estimate the AR(1) coefficient of the temporal covariance, we employ the method presented by Kay in (22). For more details, interested readers are encouraged to refer to our technical report (23).

\section{Results}

We perform two studies to analyze the effect of different covariance structures on the calibration session length and classification accuracy, respectively.

\subsection{Study 1: Analysis of Required Calibration Session Length}

Sample size required to achieve an invertible covariance estimation with a desired estimation accuracy is an indication of the calibration session length for EEG-based BCI systems. As the length of the calibration length increases, more samples are collected and better parameter estimations are obtained. However, as explained above, due to practical reasons for BCI operation, shorter calibration sessions are preferred. Using Monte Carlo simulations on synthetic data and computing the Cramer-Rao bound on covariance estimation error, we performed an error-calibration length analysis for the following Kronecker Product covariance structures with full spatial covariances and different temporal covariances: (i) full (GKS), (ii) Toeplitz (KST), and (iii) identity (KSI) temporal covariance matrices. Assume that $n_{c h} \times 1$ vector $\boldsymbol{\theta}^{\text {ch }}$ and $n_{t} \times 1$ vector $\boldsymbol{\theta}^{t}$ are the vectorized parameters of the spatial and temporal covariances respectively. Then define:

$$
\boldsymbol{\Gamma}_{\mathbf{0}}=\left(\boldsymbol{\theta}^{t} \otimes \mathbb{I}_{n t} \quad \mathbb{I}_{n_{c h}} \otimes \boldsymbol{\theta}^{c h}\right)
$$

where $\mathbb{I}_{m}$ is the identity matrix of size $(m \times m)$. Then If the full covariance matrix could be represented as a linear function of the parameters, one can define a mapping matrix $\mathbf{P}$ such that:

$$
\operatorname{vec}\left\{\boldsymbol{\Sigma}_{t} \otimes \boldsymbol{\Sigma}_{c h}\right\}=\mathbf{P} \cdot \operatorname{vec}\left\{\boldsymbol{\theta}^{t} \cdot \boldsymbol{\theta}^{c h}\right\}
$$


Accordingly, the Cramer-Rao bound is computed from (24):

$$
C R B=\mathbf{P} \boldsymbol{\Gamma}_{0}\left(\boldsymbol{\Gamma}_{0}^{T} \mathbf{P}^{*}\left(\boldsymbol{\Sigma}^{-T} \otimes \boldsymbol{\Sigma}^{-1}\right) \mathbf{P} \boldsymbol{\Gamma}_{0}\right)^{\dagger} \boldsymbol{\Gamma}_{0}^{T} \mathbf{P}^{*}
$$

We compute the RMSE as function of the sample size for three different covariances structures: (i) full temporal covariance (GKS), (ii) Toeplitz temporal covariance (KST) and (iii) Identity temporal covariance (KSI).We fix three true covariance values, one from each structure and during Monte Carlo simulations we generate data by randomly choosing among these three covariance structures. Every data generated in such a random fashion is then used to estimate the covariances under three models. For each sample size the number of times we generate different data from each chosen covariance is $N_{R}=5000$. CRBs for each model under the chosen true covariance structures are also computed. We denote the underlying true covariances as $\boldsymbol{\Sigma}_{r}$ for $r=1,2,3$. Note that for each $r$, we keep the "true covariance" constant while comparing models with each other using the same synthetically generated data. Here $\widehat{\boldsymbol{\Sigma}}_{k}$ is the estimated full covariance at simulation step $k, \boldsymbol{\Sigma}_{r}$ is the true covariance at Monte Carlo run $r,\|\cdot\|_{F}$ is the Frobenius norm; and $N_{R}$ is the total number of Monte Carlo simulations. For these simulations, we assume that the number of the channels and number of time samples are equal such that $N_{t}=N_{c h}=4$. The RMSE is calculated as below.

$$
R M S E=\frac{1}{3} \sum_{r=1}^{3} \sqrt{\frac{1}{N_{R}} \sum_{k=1}^{N_{R}} \frac{\left\|\boldsymbol{\Sigma}_{r}-\widehat{\boldsymbol{\Sigma}}_{k}\right\|_{F}^{2}}{\left\|\boldsymbol{\Sigma}_{r}\right\|_{F}^{2}}}
$$

Moreover, using the CRB on covariance estimation error, we compute a lower bound on the RMSE as

$$
L B=\sqrt{\frac{\operatorname{tr}\{C R B\}}{N \cdot\left\|\boldsymbol{\Sigma}_{r}\right\|_{F}^{2}}}
$$

where $N$ is the sample size.

Simulation results for root-MSE and LB are presented in 1. From this figure, we observe that for a small sample size, which corresponds to shorter calibration length, the estimation error is the smallest under KSI. However, as calibration length increases the estimation error is smallest for the model with a higher complexity. We think that when the number of samples is low there is higher variance introduced to the estimation error from more 
complex covariance structure models. However, as the number of samples increases this variance decreases, and the model with the least amount of complexity introduces more bias. Especially this is the case in our simulations because a full Kronecker product structure, GKS, is the asymptotically unbiased estimator for all the proposed true structures. This analysis can be used as an indication of the calibration session design and indicate us when to switch from one model to another.

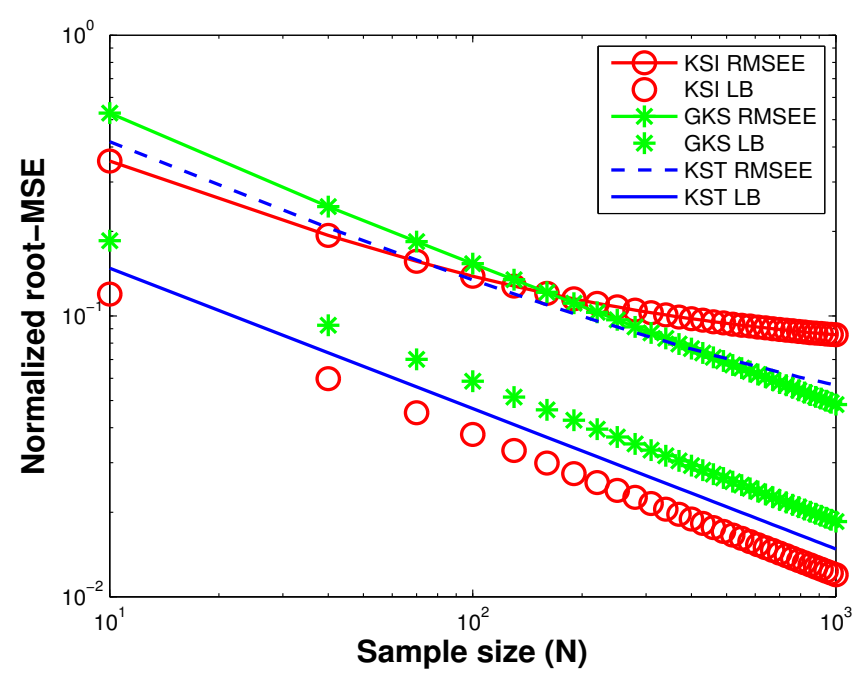

Figure 1: Normalized root-MSE and LB as a function of the sample size for three different covariance structures.

5.2. Study 2: Effect of different covariance structures on the EEG-based BCI classification performance

For this study, we collected data from 12 healthy participants using the language-model-assisted EEG-based typing BCI, RSVPKeyboard ${ }^{\mathrm{TM}}$, to assess the effect of different covariance structures on the BCI user intent inference, namely classification performance.

\subsubsection{Data Collections}

Calibration data were collected from 12 healthy users who had consented to participate according to the IRB-approved protocol (IRB130107) (3). Each user performed 12 calibration sessions for all possible combinations 
of 4 inter trial interval (ITI) values, which is defined as the time interval between consecutive trials, $(\{200 ; 150 ; 100 ; 85\} \mathrm{ms})$ and 3 presentation paradigms (RCP, SCP and RSVP) as described in Section 2.1.

EEG data were collected according to the International 10/20 EEG configuration from 16 EEG locations: Fp1, Fp2, F3, F4, Fz, Fc1, Fc2, Cz, P1, P2, $\mathrm{C} 1, \mathrm{C} 2, \mathrm{Cp} 3, \mathrm{Cp} 4, \mathrm{P} 5$ and $\mathrm{P} 6$. The sampling frequency for those recording was set to $256 \mathrm{~Hz}$ and the recorded signals were band-pass filtered with the pass-band of $[1.5,42] \mathrm{Hz}$. The preprocessed data was down-sample by order of 2. The EEG signal recorded from multiple channels was windowed in a time window of $[0,500) \mathrm{ms}$, from the onset of every stimulus, and assigned to that trial as its EEG response. Finally the windowed data from every channel was concatenated to form the feature vector $\mathbf{y}^{i}$ for trial $i^{\text {th }}$ as we defined in equation (3) in section 2.3. For every trial $N_{t}=64$ and $N_{c h}=16$.

\subsubsection{Classification performance assessment}

We calculated the area under the receiver operating characteristics (ROC) curve (AUC) values, for every calibration data using a 10-fold cross validation assuming signal models with different covariance structures. We use AUC as a classification accuracy indicator for models with four different covariance structures: (i) GKS , (ii) KST, (iii) KSI, (iv) Kronecker-product structure with $\mathrm{AR}(1)$ temporal covariance $(\operatorname{KSAR}(1))$. Moreover, we compute the class conditional non-structured (NS) full covariance matrices for every data set to be compared with the 4 structured covariance models.

For each particular ITI and presentation paradigm combination, using range bars, we employed all the calibration data and compared the median of AUC values for NS estimator, GSK, KST, KSI and KSAR(1) in Figures $2 \mathrm{a}$, 2b, and 2c. Since the number of supervised observations in every data set is proportionally lower than the number of parameters of every covariance structure, we apply RDA to regularize the estimated class conditional covariance matrices.

The results of sub-figure $2 \mathrm{a}$ show that for RSVP paradigm, the maximum performance improvement is observed at ITI $=150 \mathrm{~ms}$, which previously has been shown to provide the best performance for NS estimator among the four ITIs (3). For RSVP paradigm, and ITI $=150 \mathrm{~ms}$, one can observe a trend in median AUCs which is inversely related to the model complexity; that is, a trend of improvement in the median AUC is observed as the model order (the number of parameters to be estimated) decreases. For this presentation paradigm, although the assumed structures does not demonstrate significant 


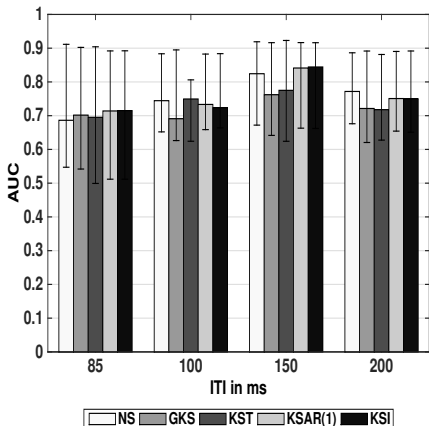

(a) RSVP

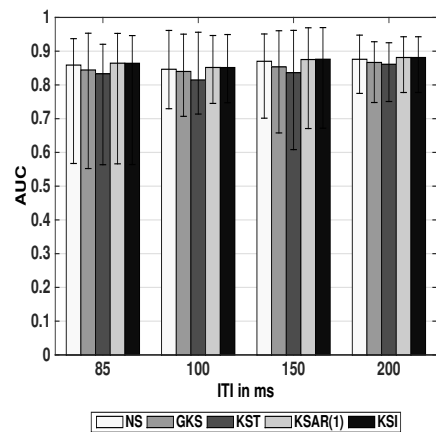

(b) SCP

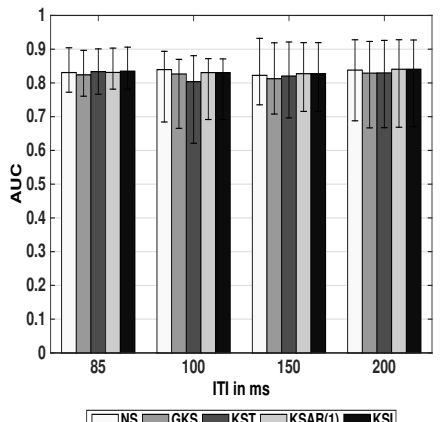

(c) $\mathrm{RCP}$

Figure 2: Bar charts of the median area under the receiver operation characteristics (ROC) curves (AUC) with bar range indicating the maximum and minimum value for twelve users calculated by use of different signal models for every presentation paradigm and ITI combinations when the classifiers are trained with all training data.

performance improvements, the models with KSAR(1) and KSI covariance structures performed equal to or better than NS estimator with lower model order complexity. That is, as the results of Section 5.1 indicate, under the models of KSAR(1) and KSI, shorter BCI calibration sessions can be designed to achieve the same performance as a model that uses no covariance structure.

Moreover, results presented in sub-figure $2 \mathrm{~b}$ illustrate a similar trend among the median AUC values obtained from signal models with different covariance structures, for all ITI values. Based on these results, for the SCP paradigm, one can improve the classification performance by use of signal models with $\operatorname{KSAR}(1)$ and KSI covariance structures.

On the other hand, unlike the other presentation paradigms, in the results presented for RCP paradigm in sub-figure 2c, we observe that all the classifiers developed based on signal models with different covariance structures perform almost similarly (similar median AUC values). This can be due to the fact that for this presentation paradigm, the number of observations in the target class is twice the number for the other presentation paradigms. The increase in the sample size can improve the performance of more complex models and relax the necessity of restricting covariance structures. However, we can still observe that classifiers built under the signal model assumptions with KSAR(1) and KSI covariance structures perform better than the other models, for most ITIs in median. 


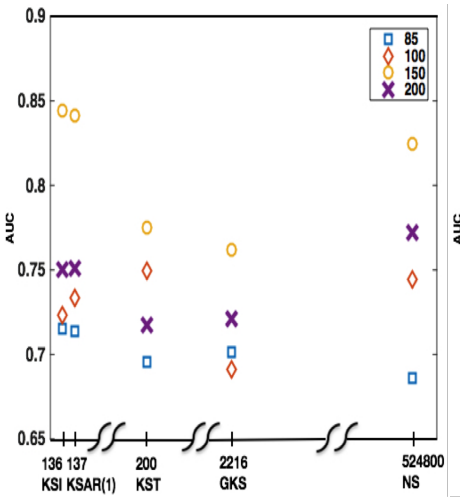

(a) RSVP

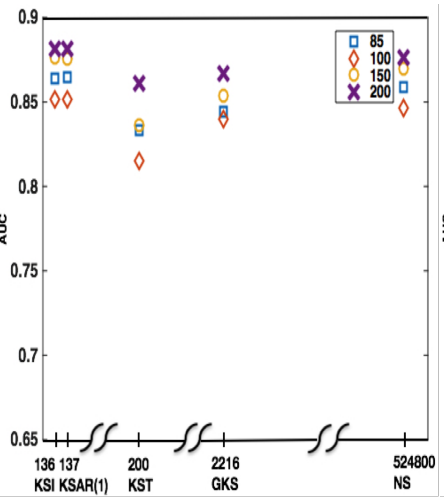

(b) SCP

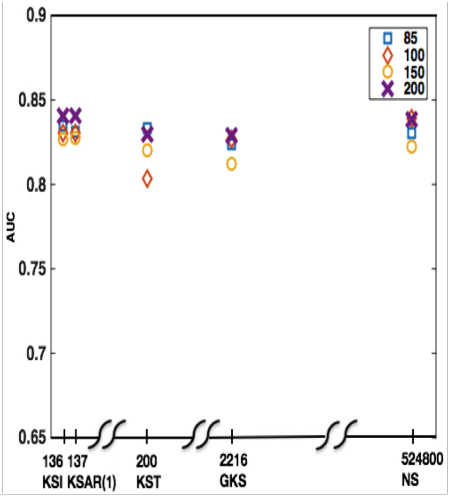

(c) $\mathrm{RCP}$

Figure 3: The median of AUC among twelve BCI users for all ITIs and presentation paradigms as a function of the model order complexity (the number of parameters to be estimated) of signal models with different covariance structures. Complexity numbers 136, 137, 200, 2216 and 524800 correspond to models with KSI, KSAR(1), KST, GKS, and non-structured (NS) covariances respectively.

Furthermore, we illustrate the AUC values of different classifiers built under different covariance structure assumptions as a function of the model order complexity (the number of covariance parameters to be estimated) in Figures $3 \mathrm{a}, 3 \mathrm{~b}$ and $3 \mathrm{c}$. As the plots suggest, the signal models with KSAR(1) and KSI covariance structures not only show a trend in the improvement of the median AUC, they also have significantly lower model complexity (the number of parameters to be estimated for KSI and KSAR(1) are 136 and 137, respectively) compared to the model that does not assume a structured covariance (the number of parameters to be estimated is 524800). As the results of Section 5.1 suggest this decrease in model complexity results in also a decrease in calibration session length, which is a very desirable feature for BCI systems that are designed for real-life applications.

Finally, we evaluate the performance of the proposed method on the system accuracy when the classifiers are trained with calibration sets of different sizes. We use this study to see how the performance of each model varies with respect to the size of the training set. As described in section 2.3 each calibration session of RSVPkeyboard ${ }^{\mathrm{TM}}$ consists of a number of sequences which are used to estimate the class conditional distributions. The number of sequences to show in calibration effects the duration of calibration session in time. It is desired that calibration sessions should be as short as possible 
to prevent user fatigue and nonstationarities in the EEG. We compute the median AUC for different calibration lengths from 12 healthy subject for every calibration data using a 10 -fold cross validation assuming signal models with different covariance structures. Here in the results section, we demonstrate the results for ITI $=150 \mathrm{~ms}$ and the results for other ITIs are added in the Appendix, see Figure 4, and Tables 1, 2, 3, 4, 5, 6, 7, 8, 9, 10, 11, 12 .

Figure 4 compares the median of AUC values for NS estimator, GSK, KST, KSI and KSAR(1) for different calibration lengths of $[10 ; 20 ; 30 ; 40$; 50] sequences for all presentation paradigms and a fixed inter trial interval (ITI) of $150 \mathrm{~ms}{ }^{1}$ using range bars. We also performed a statistical test to compare the differences among the AUC values, see Tables 1, 2 and 3. With a significance level of $\alpha=0.1$, we observe significant differences between the KSAR (1) and NS, and KSI and NS for the sequence length of 10 for the RSVP paradigm and sequence lengths of 10 and 20 for the SCP and RCP paradigms. As shown in Figure 4a in the case of the RSVP presentation paradigm the median classification AUC can reach up to $70 \%$ within 20 sequences when KSAR(1) or KSI (Identity temporal covariance matrix) models are used. On the other hand, the same level of classification performance for RDA can be achieved with at least 40 sequences. A similar trend can be observed in Figure $4 \mathrm{~b}$ such that in the case of SCP paradigm, we obtain higher AUCs with all the models, the median classification AUC can reach up to 80 $\%$ within 20 sequences when KSAR(1) or KSI models are used, while to reach the same level of classification AUC in RDA model 40 sequences are required. Moreover, as we can see in Figure 4c, in RCP paradigm with 40 sequences in KSAR(1), KSI and KST model, \% 80 accuracy could be reached while RDA (NS) model requires more than 50 sequences to reach the same level of classification accuracy. This trend shows us that it is possible to shorten the calibration session length by choosing KSI, KSAR(1) or KST covariance structures compared to the NS structure. Here the best results are obtained for SCP presentation paradigm.

Similar calibration session length improvement trend can be observed for different ITI values as shown in the Appendix Tables 4, 5, 6, 7, 8, 9, 10, 11. 12. Here we observe that when $\mathrm{ITI}=85 \mathrm{~ms}$, under SCP paradigm with significance level of $\alpha=0.1$, the AUC values for $\operatorname{KSAR}(1)$ and KSI structures

\footnotetext{
${ }^{1}$ The current RSVP Keyboard uses $150 \mathrm{~ms}$ as the optimal ITI (3)
} 


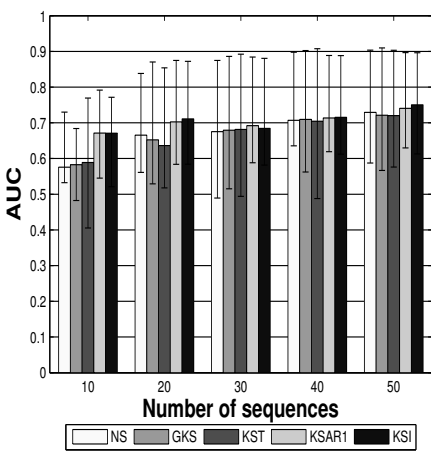

(a) RSVP

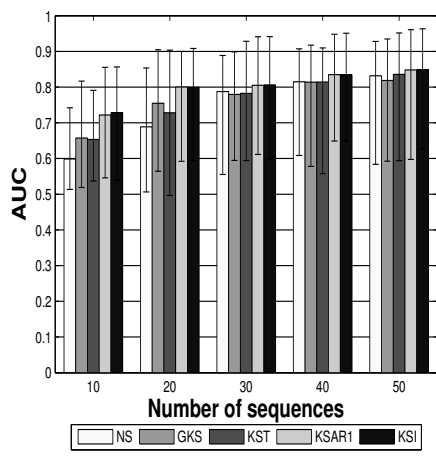

(b) SCP

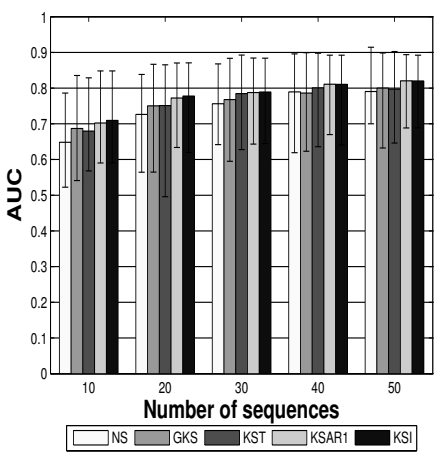

(c) $\mathrm{RCP}$

Figure 4: Bar charts of the median area under the receiver operation characteristics (ROC) curves (AUC) with bar range indicating the maximum and minimum value for twelve users calculated by use of different signal models for every presentation paradigm ITI $=150 \mathrm{~ms}$ and different calibration lengths $(\{10 ; 20 ; 30 ; 40 ; 50\}$ sequences $)$ to train the classifiers.

are significantly higher than the AUC values for NS covariance when the calibration sequence lengths are 10 and 20. When the ITI is increased to $100 \mathrm{~ms}$, for sequence lengths of 10, 20 and 30, the same trend is observed. However, when ITI $=200 \mathrm{~ms}$, the significance is observed only for the sequence length of 10. On the other hand, for the RCP paradigm, under the significance level of $\alpha=0.1$, we observe that for all the ITI values, KSI and KSAR(1) models are significantly better than the NS covariance model for sequence lengths of 10, 20 and 30. Among all the results, RSVP shows the least improvement.

\begin{tabular}{|c||c|c|c|c|c|}
\hline$S M$ v.s. $N S$ & 10 & 20 & 30 & 40 & 50 \\
\hline \hline$G K S$ v.s. $N S$ & 0.7612 & 0.4662 & 0.5338 & 0.6006 & 0.5563 \\
$K S T$ v.s. $N S$ & 0.6224 & 0.6006 & 0.5786 & 0.6851 & 0.6647 \\
$A R(1)$ v.s. $N S$ & 0.0298 & 0.1888 & 0.2388 & 0.3994 & 0.3563 \\
$K S I$ v.s. $N S$ & 0.0503 & 0.1593 & 0.3149 & 0.3563 & 0.2214 \\
\hline
\end{tabular}

Table 1: p-values of a right-sided Wilcoxon rank sum test for the different covariance models versus NS for different calibrations lengths $(\{10 ; 20 ; 30 ; 40 ; 50\}$ sequences $)$ in RSVP paradigm and ITI $150 \mathrm{~ms}$. 


\begin{tabular}{|c||c|c|c|c|c|}
\hline$S M$ v.s. $N S$ & 10 & 20 & 30 & 40 & 50 \\
\hline \hline$G K S$ v.s. $N S$ & 0.1888 & 0.3563 & 0.5113 & 0.5338 & 0.6006 \\
$K S T$ v.s. $N S$ & 0.1208 & 0.4887 & 0.5338 & 0.5786 & 0.5786 \\
$A R(1)$ v.s. $N S$ & 0.0028 & 0.0638 & 0.2214 & 0.3149 & 0.3776 \\
$K S I$ v.s. $N S$ & 0.0034 & 0.0800 & 0.2214 & 0.3776 & 0.3776 \\
\hline
\end{tabular}

Table 2: p-values of a right-sided Wilcoxon rank sum test for the different covariance models versus NS for different calibrations lengths $(\{10 ; 20 ; 30 ; 40 ; 50\}$ sequences $)$ in SCP paradigm and ITI $150 \mathrm{~ms}$.

\begin{tabular}{|c||c|c|c|c|c|}
\hline$S M$ v.s. $N S$ & 10 & 20 & 30 & 40 & 50 \\
\hline \hline$G K S$ v.s. $N S$ & 0.1888 & 0.2388 & 0.2949 & 0.5786 & 0.5338 \\
$K S T$ v.s. $N S$ & 0.2388 & 0.2949 & 0.2048 & 0.5113 & 0.4887 \\
$A R(1)$ v.s. $N S$ & 0.0298 & 0.0638 & 0.1737 & 0.2388 & 0.3149 \\
$K S I$ v.s. $N S$ & 0.0259 & 0.0568 & 0.1737 & 0.2388 & 0.3563 \\
\hline
\end{tabular}

Table 3: p-values of a right-sided Wilcoxon rank sum test for the different covariance models versus NS for different calibrations lengths $(\{10 ; 20 ; 30 ; 40 ; 50\}$ sequences) in RCP paradigm and ITI $150 \mathrm{~ms}$.

\section{Conclusions}

In this paper, we developed signal models for multichannel EEG, leading to different covariance structures for class-conditional densities of multivariate EEG features to be used in intent inference engines for EEG-based brain computer interfaces. We showed that under certain stationarity assumptions, concatenated EEG feature vectors follow a multivariate Gaussian distribution with a Kronecker product structure, which factorizes into spatial and temporal covariance matrices. We also showed that different temporal and independence assumptions on temporal propagation of signals result in further structures on temporal covariance.

We performed two studies, one with synthetic and one with real EEG data collected through a letter-by-letter typing BCI to analyze the effect of the structured covariance models for EEG on BCI calibration session length and user intent detection accuracy, respectively. Our results of the first study show that as the model order complexity decreases (stronger assumptions on the covariance structure resulting in lower degrees of freedom in covariance) the session length could be shortened compared to a signal model that assumes no specific covariance structure, while it achieves similar covariance es- 
timation accuracy. The results of the second study show that the models with structured covariance not only show a trend in improvement in user intent detection accuracy, but also have significantly lower model order complexity compared to a signal model with no specific covariance structure. These results implies that more structured covariances could be used to potentially shorten BCI calibration sessions, while preserving system performance.

In future work, we will extend our approaches to relax the stationarity assumptions and select the model orders optimally to more realistically model the EEG signals. We will also investigate further structure constraints on head models in forward linear source model to represent different spatial covariance structures. Appropriately structured Gaussian covariance models, coupled with growing EEG data from the same user over time, and from multiple users across the user population could result in significantly improved BCI system performance by statistical modeling the multichannel EEG within the BCI contexts.

\section{Acknowledgment}

This work is supported by NIH 2R01DC009834, NIDRR H133E140026, NSF CNS-1136027, IIS-1118061, IIS-1149570, CNS-1544895, SMA-0835976.

For supplemental materials, please visit http://hdl.handle.net/2047/D20199232 for the CSL Collection in the Northeastern University Digital Repository System.

[1] M. Akcakaya, B. Peters, M. Moghadamfalahi, A. Mooney, U. Orhan, B. Oken, D. Erdogmus, M. Fried-Oken, Noninvasive brain computer interfaces for augmentative and alternative communication, IEEE Reviews in Biomedical Engineering 7 (1) (2014) 31-49. doi:10.1109/ RBME. 2013.2295097.

[2] L. A. Farwell, E. Donchin, Talking off the top of your head: toward a mental prosthesis utilizing event-related brain potentials, Electroencephalography and clinical Neurophysiology 70 (6) (1988) 510-523.

[3] M. Moghadamfalahi, U. Orhan, M. Akcakaya, H. Nezamfar, M. FriedOken, D. Erdogmus, Language-model assisted brain computer interface for typing: A comparison of matrix and rapid serial visual presentation, IEEE Transactions on Neural Systems and Rehabilitation Engineering PP (99) (2015) 1-1. doi:10.1109/TNSRE. 2015.2411574. 
[4] L. Acqualagna, M. S. Treder, M. Schreuder, B. Blankertz, A novel brain-computer interface based on the rapid serial visual presentation paradigm, in: Proceedings of EMBC, Vol. 1, 2010, pp. 2686-2689.

[5] U. Orhan, K. E. Hild, D. Erdogmus, B. Roark, B. Oken, M. Fried-Oken, Rsvp keyboard: An eeg based typing interface, in: International Conference on Acoustics, Speech and Signal Processing (ICASSP), IEEE, 2012, pp. 645-648.

[6] E. E. Sutter, The brain response interface: Communication through visually-induced electrical brain responses, J. Microcomput. Appl. 15 (1) (1992) 31-45. doi:10.1016/0745-7138(92)90045-7. URL http://dx.doi.org/10.1016/0745-7138(92)90045-7

[7] H. Nezamfar, U. Orhan, D. Erdogmus, K. Hild, S. Purwar, B. Oken, M. Fried-Oken, On visually evoked potentials in eeg induced by multiple pseudorandom binary sequences for brain computer interface design, in: Acoustics, Speech and Signal Processing (ICASSP), 2011 IEEE International Conference on, 2011, pp. 2044-2047. doi:10.1109/ICASSP. 2011.5946914.

[8] M. Xu, H. Qi, B. Wan, T. Yin, Z. Liu, D. Ming, A hybrid bci speller paradigm combining p300 potential and the ssvep blocking feature, Journal of Neural Engineering 10 (2) (2013) 026001.

[9] R. A. Davis, Gaussian Process, John Wiley Sons, Ltd, 2006. doi:10. 1002/9780470057339.vag002.

URL http://dx .doi .org/10.1002/9780470057339.vag002

[10] C. E. Rasmussen, C. K. I. Williams, Gaussian Processes for Machine Learning (Adaptive Computation and Machine Learning), The MIT Press, 2005.

[11] F. J. M. Jr., The kolmogorov-smirnov test for goodness of fit, Journal of the American Statistical Association 46 (253) (1951) 68-78.

[12] D. B. Ryan, G. E. Frye, G. Townsend, D. R. Berry, S. Mesa-G, N. A. Gates, E. W. Sellers, Predictive spelling with a p300-based braincomputer interface: Increasing the rate of communication, International Journal of Human-Computer Interaction 27 (1) (2010) 69-84. 
[13] B. Blankertz, F. Losch, M. Krauledat, G. Dornhege, G. Curio, K.R. Muller, The berlin brain-computer interface: Accurate performance from first-session in bci-naïve subjects, Biomedical Engineering, IEEE Transactions on 55 (10) (2008) 2452-2462. doi:10.1109/TBME. 2008. 923152

[14] M. Moghadamfalahi, U. Orhan, M. Akcakaya, D. Erdogmus, Bayesian Priors for Classifier Design in RSVP Keyboard.

[15] M. S. Treder, B. Blankertz, (C)overt attention and visual speller design in an ERP-based brain-computer interface., Behavioral and brain functions : BBF 6 (1) (2010) 28. doi:10.1186/1744-9081-6-28.

URL http://www.behavioralandbrainfunctions.com/content/6/ $1 / 28$

[16] P. Stoica, M. Viberg, Maximum likelihood parameter and rank estimation in reduced-rank multivariate linear regressions, Signal Processing, IEEE Transactions on 44 (12) (1996) 3069-3078. doi:10.1109/78. 553480

[17] O. Ledoit, M. Wolf, Improved estimation of the covariance matrix of stock returns with an application to portfolio selection, Journal of Empirical Finance 10 (5) (2003) 603 - 621.

[18] U. Orhan, D. Erdogmus, B. Roark, B. Oken, M. Fried-Oken, Offline analysis of context contribution to erp-based typing bci performance, Journal of Neural Engineering 10 (6) (2013) 066003.

[19] J. H. Friedman, Regularized discriminant analysis, Journal of the American statistical association 84 (405) (1989) 165-175.

[20] U. Orhan, RSVP keyboard : an EEG based BCI typing system with context information fusion.

[21] N. Lu, D. Zimmerman, On the Likelihood-based Inference for a Separable Covariance Matrix, Tech. Rep. 337, Department Of Statistics and Actuarial Science, Univ. of Iowa, Iowa City, Iowa (2004).

[22] S. Kay, Recursive maximum likelihood estimation of autoregressive processes, Acoustics, Speech and Signal Processing, IEEE Transactions on 31 (1) (1983) 56-65. doi:10.1109/TASSP. 1983.1164050. 
[23] P. Gonzalez-Navarro, Spatio-temporal EEG Models for BCI, Tech. rep., Technical Report, BSPIRAL-150724-R0, Northeastern University (2015).

URL http://hdl.handle.net/2047/d20194049

[24] K. Werner, M. Jansson, P. Stoica, On Estimation of Covariance Matrices With Kronecker Product Structure 56 (2) (2008) 478-491.

\section{Appendix}

\begin{tabular}{|c||c|c|c|c|c|}
\hline$S M$ v.s. $N S$ & 10 & 20 & 30 & 40 & 50 \\
\hline \hline$G K S$ v.s. $N S$ & 0.4662 & 0.2949 & 0.4662 & 0.5113 & 0.4437 \\
$K S T$ v.s. $N S$ & 0.6647 & 0.2949 & 0.3563 & 0.5113 & 0.4214 \\
$A R(1)$ v.s. $N S$ & 0.1328 & 0.2048 & 0.2949 & 0.3563 & 0.2949 \\
$K S I$ v.s. $N S$ & 0.1593 & 0.2048 & 0.2756 & 0.3149 & 0.2949 \\
\hline
\end{tabular}

Table 4: p-values of a right-sided Wilcoxon rank sum test for the different covariance models versus NS for different calibrations lengths $(\{10 ; 20 ; 30 ; 40 ; 50\}$ sequences $)$ in RSVP paradigm and ITI $85 \mathrm{~ms}$.

\begin{tabular}{|c||c|c|c|c|c|}
\hline$S M$ v.s. $N S$ & 10 & 20 & 30 & 40 & 50 \\
\hline \hline$G K S$ v.s. $N S$ & 0.4662 & 0.3353 & 0.3353 & 0.6006 & 0.5338 \\
$K S T$ v.s. $N S$ & 0.3149 & 0.2388 & 0.5786 & 0.4214 & 0.7244 \\
$A R(1)$ v.s. $N S$ & 0.0194 & 0.0989 & 0.1208 & 0.1737 & 0.2214 \\
$K S I$ v.s. $N S$ & 0.0444 & 0.0716 & 0.1457 & 0.2048 & 0.2214 \\
\hline
\end{tabular}

Table 5: p-values of a right-sided Wilcoxon rank sum test for the different covariance models versus NS for different calibrations lengths $(\{10 ; 20 ; 30 ; 40 ; 50\}$ sequences $)$ in SCP paradigm and ITI $85 \mathrm{~ms}$. 


\begin{tabular}{|c||c|c|c|c|c|}
\hline$S M$ v.s. $N S$ & 10 & 20 & 30 & 40 & 50 \\
\hline \hline$G K S$ v.s. $N S$ & 0.0989 & 0.2214 & 0.2388 & 0.4662 & 0.4887 \\
$K S T$ v.s. $N S$ & 0.1095 & 0.2949 & 0.4887 & 0.2756 & 0.4437 \\
$A R(1)$ v.s. $N S$ & 0.0028 & 0.0121 & 0.0225 & 0.0638 & 0.2388 \\
$K S I$ v.s. $N S$ & 0.0015 & 0.0060 & 0.0166 & 0.0503 & 0.2048 \\
\hline
\end{tabular}

Table 6: p-values of a right-sided Wilcoxon rank sum test for the different covariance models versus NS for different calibrations lengths $(\{10 ; 20 ; 30 ; 40 ; 50\}$ sequences $)$ in RCP paradigm and ITI $85 \mathrm{~ms}$.

\begin{tabular}{|c||c|c|c|c|c|}
\hline$S M$ v.s. $N S$ & 10 & 20 & 30 & 40 & 50 \\
\hline \hline$G K S$ v.s. $N S$ & 0.3563 & 0.2214 & 0.3353 & 0.5563 & 0.5113 \\
$K S T$ v.s. $N S$ & 0.1737 & 0.3563 & 0.4437 & 0.6006 & 0.4214 \\
$A R(1)$ v.s. $N S$ & 0.1457 & 0.1208 & 0.1737 & 0.3563 & 0.2569 \\
$K S I$ v.s. $N S$ & 0.0891 & 0.0891 & 0.1737 & 0.3353 & 0.2214 \\
\hline
\end{tabular}

Table 7: p-values of a right-sided Wilcoxon rank sum test for the different covariance models versus NS for different calibrations lengths $(\{10 ; 20 ; 30 ; 40 ; 50\}$ sequences $)$ in RSVP paradigm and ITI $100 \mathrm{~ms}$.

\begin{tabular}{|c||c|c|c|c|c|}
\hline$S M$ v.s. $N S$ & 10 & 20 & 30 & 40 & 50 \\
\hline \hline$G K S$ v.s. $N S$ & 0.0503 & 0.3994 & 0.0800 & 0.2756 & 0.3149 \\
$K S T$ v.s. $N S$ & 0.0390 & 0.3994 & 0.1457 & 0.6006 & 0.5113 \\
$A R(1)$ v.s. $N S$ & 0.0001 & 0.0716 & 0.0298 & 0.1457 & 0.1593 \\
$K S I$ v.s. $N S$ & 0.0003 & 0.0716 & 0.0298 & 0.1328 & 0.2048 \\
\hline
\end{tabular}

Table 8: p-values of a right-sided Wilcoxon rank sum test for the different covariance models versus NS for different calibrations lengths $(\{10 ; 20 ; 30 ; 40 ; 50\}$ sequences $)$ in SCP paradigm and ITI $100 \mathrm{~ms}$.

\begin{tabular}{|c||c|c|c|c|c|}
\hline$S M$ v.s. $N S$ & 10 & 20 & 30 & 40 & 50 \\
\hline \hline$G K S$ v.s. $N S$ & 0.1208 & 0.2569 & 0.5563 & 0.5563 & 0.5113 \\
$K S T$ v.s. $N S$ & 0.0444 & 0.3149 & 0.5113 & 0.4662 & 0.4887 \\
$A R(1)$ v.s. $N S$ & 0.0028 & 0.0342 & 0.1208 & 0.2388 & 0.1593 \\
$K S I$ v.s. $N S$ & 0.0034 & 0.0568 & 0.1593 & 0.3149 & 0.2214 \\
\hline
\end{tabular}

Table 9: p-values of a right-sided Wilcoxon rank sum test for the different covariance models versus NS for different calibrations lengths $(\{10 ; 20 ; 30 ; 40 ; 50\}$ sequences $)$ in RCP paradigm and ITI $100 \mathrm{~ms}$. 


\begin{tabular}{|c||c|c|c|c|c|}
\hline$S M$ v.s. $N S$ & 10 & 20 & 30 & 40 & 50 \\
\hline \hline$G K S$ v.s. $N S$ & 0.7051 & 0.4437 & 0.4887 & 0.6006 & 0.6224 \\
$K S T$ v.s. $N S$ & 0.9702 & 0.6437 & 0.6006 & 0.6851 & 0.8263 \\
$A R(1)$ v.s. $N S$ & 0.0259 & 0.1888 & 0.2388 & 0.2756 & 0.2388 \\
$K S I$ v.s. $N S$ & 0.0390 & 0.2949 & 0.2214 & 0.2949 & 0.2569 \\
\hline
\end{tabular}

Table 10: p-values of a right-sided Wilcoxon rank sum test for the different covariance models versus NS for different calibrations lengths $(\{10 ; 20 ; 30 ; 40 ; 50\}$ sequences $)$ in RSVP paradigm and ITI $200 \mathrm{~ms}$.

\begin{tabular}{|c||c|c|c|c|c|}
\hline$S M$ v.s. $N S$ & 10 & 20 & 30 & 40 & 50 \\
\hline \hline$G K S$ v.s. $N S$ & 0.4437 & 0.4887 & 0.5113 & 0.6006 & 0.4662 \\
$K S T$ v.s. $N S$ & 0.5113 & 0.6006 & 0.5338 & 0.5786 & 0.6851 \\
$A R(1)$ v.s. $N S$ & 0.0194 & 0.1593 & 0.2048 & 0.3149 & 0.2214 \\
$K S I$ v.s. $N S$ & 0.0225 & 0.0891 & 0.2569 & 0.2949 & 0.2569 \\
\hline
\end{tabular}

Table 11: p-values of a right-sided Wilcoxon rank sum test for the different covariance models versus NS for different calibrations lengths $(\{10 ; 20 ; 30 ; 40 ; 50\}$ sequences $)$ in SCP paradigm and ITI $200 \mathrm{~ms}$.

\begin{tabular}{|c||c|c|c|c|c|}
\hline$S M$ v.s. $N S$ & 10 & 20 & 30 & 40 & 50 \\
\hline \hline$G K S$ v.s. $N S$ & 0.0800 & 0.2569 & 0.3563 & 0.4662 & 0.6224 \\
$K S T$ v.s. $N S$ & 0.6437 & 0.2214 & 0.6224 & 0.5338 & 0.4887 \\
$A R(1)$ v.s. $N S$ & 0.0072 & 0.0166 & 0.0989 & 0.1888 & 0.1888 \\
$K S I$ v.s. $N S$ & 0.0041 & 0.0342 & 0.1208 & 0.2048 & 0.1888 \\
\hline
\end{tabular}

Table 12: p-values of a right-sided Wilcoxon rank sum test for the different covariance models versus NS for different calibrations lengths $(\{10 ; 20 ; 30 ; 40 ; 50\}$ sequences $)$ in RCP paradigm and ITI $200 \mathrm{~ms}$. 